\title{
Corrigenda
}

\section{Transformational Methods and Their Application to Complexity Problems}

\author{
Burkhard Monien \\ Fachbereich Mathematik/Informatik der Gesamthochschule Paderborn, \\ Warburger Str. 100, D-4790 Paderborn, Germany (Fed. Rep.) \\ Acta Informatica 6, 95-108 (1976)
}

The proof of Lemma 4 (p. 105) is not correct and therefore also the proof of the main theorem of Section 4 (Theorem 8) does not hold. We will give here a new proof of Theorem 8 . In order to do so replace Lemma 4 and Theorem 6 by a new Theorem 6:

Let $\Sigma$ be some alphabet; let $f_{\Sigma, v}, v \in \mathbb{N}$, be the functions defined in Section 2 and let $k, j \in \mathbb{N}, j \geqq 2$, be arbitrary numbers. Then

$$
\begin{aligned}
& L \in D_{k \cdot j+1} \Rightarrow f_{\Sigma, k}(L) \in D_{j+1}, \\
& f_{\Sigma, j+1}(L) \in D_{j} \Rightarrow f_{\Sigma, j}(L) \in D_{j+1}
\end{aligned}
$$

holds for every language $L \subset \Sigma^{*}$.

This theorem is proved with methods which are also used in the proofs of Theorem 2 and Theorem 7.

New Proof of Theorem 8. $\left(D_{j} \varsubsetneqq D_{j+1}\right.$ for all $\left.j \in \mathbb{N}\right)$.

$D_{1}$ is the class of regular sets and therefore $\left\{a^{n} b^{n} \mid n \in \mathbb{N}\right\} \notin D_{1}$. It is obvious that $\left\{a^{n} b^{n} \mid n \in \mathbb{N}\right\} \in D_{2}$ and this implies $D_{1} \subsetneq D_{2}$.

Now let us assume that $j \geqq 2$ and $D_{j}=D_{j+1}$ and choose some $L \in D_{(j+1) \cdot j+1}$. Then because of Theorem 6 and Theorem 7 the following implications hold:

$$
L \in D_{(j+1) \cdot j+1} \Rightarrow f_{j+1}(L) \in D_{j+1}=D_{j} \Rightarrow f_{j}(L) \in D_{j+1}=D_{j} \Rightarrow L \in D_{j^{2}} .
$$

This implies $D_{j^{2}+j+1}=D_{j^{2}}$ which is a contradiction to the result of O. H. Ibarra.

This method can be applied also to get hierarchy results for other types of multihead automata. Especially it can be shown in the same way that $N_{j}$ 皮 $N_{j+1}$ for all $j \in \mathbb{N}$. This sharpens a result of J.I. Seiferas ["Relating refined space complexity classes". J. Computer System Sci. 14, 100-129 (1977)]. 
The author would like to take this opportunity to mention that the first statement of Theorem 4 (NTAPE $(\log n)=$ Tape $(\log n) \Leftrightarrow C \subset$ Tape $(\log n))$ was also found independently by 1 . H.Sudborough ("On tape-bounded complexity classes and multihead finite automata". J. Computer System Sci. 10, 62-76 (1975)) and by Z. Galil ("Two way deterministic pushdown automaton languages and some open problems in the theory of computation". IEEE Conference Record 15th Annual Symposium on Switching and Automata Theory, 1974, pp. 170-177). Furthermore the author wants to thank J. Seiferas and Z. Galil for their valuable criticism of the earlier version of the proof of Theorem 8 .

Received May 23, 1977 